\title{
Usability of Tilted Plasmon Antenna with Structured Light
}

\author{
Rafael Quintero-Torres ${ }^{1 *}$, Jorge Luis Domínguez-Juárez ${ }^{1,2}$, Mariia Shutova $^{3}$, and Alexei V. Sokolov ${ }^{3}$ \\ 1 Centro de Física Aplicada y Tecnología Avanzada, Universidad Nacional Autónoma de México, Juriquilla, \\ Querétaro, 76230, México. rquintero@fata.unam.mx \\ 2 Cátedras CONACyT, CFATA, UNAM, Juriquilla, Querétaro 76230, México. jldominguezju@conacyt.mx \\ 3 Institute for Quantum Science and Engineering, Department of Physics and Astronomy, Texas A\&M Uni- \\ versity, College Station, TX 77843-4242, USA. mariia.shutova@gmail.com; sokol@tamu.edu, \\ * Correspondence: rquintero@fata.unam.mx
}

\begin{abstract}
We study the effect of oblique illumination on the functioning of a plasmonic nanoantenna for chiral light. The antenna is designed to receive a structured beam of light and produce a nanosized near-field distribution that possesses non-zero orbital angular momentum. The design consists of metal (gold) micro-rods laid on a dielectric surface and is compatible with well-developed nanofabrication techniques. Experimental arrangements often require such an antenna to operate in a tilted geometry, where input light is incident on the antenna at an oblique angle. We analyze the limitations that the angled illumination imposes and discuss approaches to mitigate these limitations. Through our numerical simulations, we find that tilt angles require modifications to the antenna design. Our analysis guides current and future experimental configurations to pushing the limits of resolution and sensitivity.
\end{abstract}

Keywords: Plasmonic nanoantenna; Electric field norm; Tilted light incidence

\section{Introduction}

Technologies that use electromagnetic fields can be used with a large frequency bandwidth, only limited by the frequency of the field used [1]. At the same time, their spatial resolution is limited by diffraction. Similarly, technologies that use electronic transport have a very small spatial resolution, limited by quantum confinement, which can reach values around one nanometer [2]. However, they have a limited frequency bandwidth, defined by electron mobility and device size. Electric field confinement by plasmonic interaction has been explored for some time with very important results, from localized light [3] to nano sources of radiation [4]. Furthermore, structured beams have been applied to increase the amount of encoded information [5], [6]; their use in conjunction with plasmonics nanoantennas is of particular interest and will result in confined field structures with sizes well beyond the diffraction limit [7].

A nanoantenna can control the field intensity of simple fields and linear states of polarization. Controlling the spatially structured field with an orbital angular momentum (OAM) of a beam of light proves more challenging. This control is anticipated to reach the dimensions defined by the beam, the antenna, and the geometrical arrangement between them. Studies show that light with orbital angular momentum can interact with single molecules and ions and directly induce quadrupole transitions [8], [9]. For efficient interaction with single molecules, light with OAM needs to be brought to the size of the interaction particle (nanometers). Plasmonic nanoantennas are the easiest way to break the diffraction limit and "focus" the OAM beam to the nanoscale. The theory for subdiffraction focusing and probing quadrupole transitions is being developed rapidly [10]. However, experimental work has been limited by some technical aspects. 
In this paper, we studied the effect of oblique illumination and simulating defocusing and adjustments close to centre on the functioning of a plasmonic nanoantenna for chiral light. We consider a nanoantenna (eight-arms) for a beam of light with OAM that is incident at an angle greater than zero, to the normal antenna plane. To gain some intuition and visualization with a case of normal incidence and Laguerre $m=1$ beam, we constructed a two-arms dipole antenna to produce a basic guide for resonance at a wavelength approximately. After, we show how to improve illumination with oblique incidence and defocusing with nanometer steps adjustments close to the centre of the normalaxis reference. We show that the electric field magnitude over the antenna, because Laguerre-Gauss (LG) beams maximum gets further from the center increasing the beam diameter, though the working frequency decreases for the same reason, and the arm length and central gap structure in antennas defines the resonant frequency. In a multi-rod antenna, attempts to correct the slanted angle $\alpha$ are limited because the antenna sees a beam growing from 1 to $1 / \cos (\alpha)$ in the two normal axes.

We considered the situation when the antenna is used in combination with an atomic force microscope (AFM) with the side-illumination objective. The angle at which the side objective is situated differs significantly from normal illumination, so it will affect the functioning of the nanoantenna, and therefore it needs to be considered in our calculations. The antenna used in this work was inspired by a geometry achievable with a commercially focused ion beam (FIB) [14]. Figure 1 is a schematic figure of a Laguerre-Gauss beam of light with OAM, the eight-arm antenna geometry, and finally, we show the intensity distribution of such beam at normal incidence.

\section{Orbital Angular Momentum}

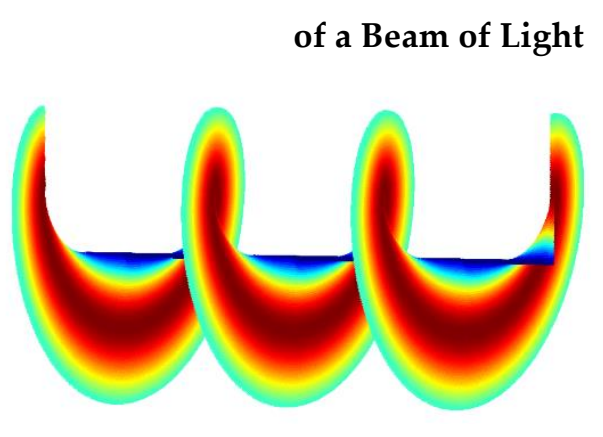

(a)

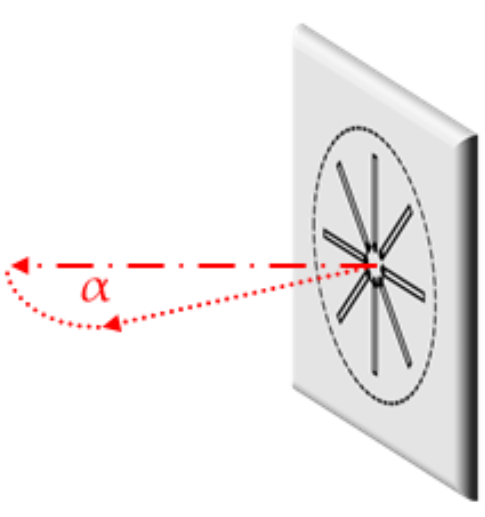

(b)

\section{Eight Arms Antenna}

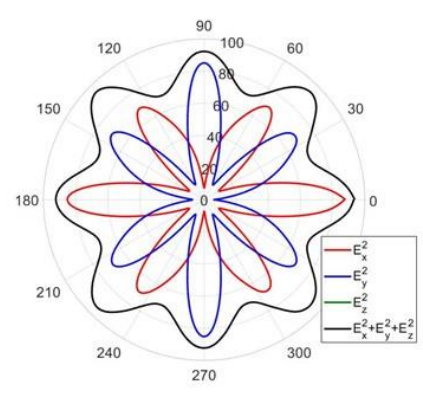

(c)

Figure 1. (a) Spatially-structured-field with an orbital angular momentum (OAM) of a beam of light (b) Antenna geometry and antenna plane angle $(\alpha)$ relative to the electromagnetic wave propagation direction. (c) Antenna electric field squared at normal incidence.

\section{Materials and Methods}

\subsection{Orbital Angular Momentum (OAM) of a beam of light}

A Laguerre-Gauss (LG) laser beam has more degrees of freedom than a regular Gaussian beam [11]. Its propagation modes have rotational symmetry along its propagation axis and carry an intrinsic rotational orbital angular momentum of ih per photon. This means that a refractive object placed along the propagation axis will experience a torque. Figure $2 \mathrm{a}$ presents a standard electric field norm $(\operatorname{norm}(E) \equiv\|E\|=$ $\left.\sqrt{\left|E_{x}\right|^{2}+\left|E_{y}\right|^{2}+\left|E_{z}\right|^{2}}=\sqrt{E_{x} E_{x}^{*}+E_{y} E_{y}^{*}+E_{z} E_{z}^{*}}\right)$ as presented by the scalar field in equation 1 . 


$$
\begin{gathered}
E(r, \varphi, z)= \\
E_{0}\left(\frac{w_{0}}{w(z)}\right)\left(\frac{\sqrt{2} r}{w(z)}\right)^{m} \exp \left(-\left(\frac{r}{w(z)}\right)^{2}\right) \exp \left(-i k z \frac{\left(r w_{0}\right)^{2}}{2\left(w(z) z_{0}\right)^{2}}\right) \exp (-i(k z+ \\
\left.\left.m \varphi(x, y)-(m+1) \tan ^{-1} \frac{z}{z_{0}}\right)\right),
\end{gathered}
$$

In this representation, a typical connection between Cartesian and cylindrical coordinates is used, $r(x, y)=\sqrt{x^{2}+y^{2}}, \varphi(x, y)=\tan ^{-1} \frac{y}{x^{\prime}}$ and with a beam radius at focus defined by $w_{0}$, a beam size parameter $w(z)=w_{0} \sqrt{1+\left(\frac{z}{z_{0}}\right)^{2}}$ and a Rayleigh range $z_{0}=$ $\frac{\pi w_{0}^{2}}{\lambda}, k$ is the wave vector and $\lambda$ is the wavelength.

The vector field propagating in the $z$-direction and with circular polarization is described with the usual Cartesian coordinates in the next expression:

$$
\vec{E}=\frac{E(r, \varphi, z)}{\sqrt{2}}\left(\hat{\imath} e^{+\frac{i \pi}{4}}+\hat{\jmath} e^{-\frac{i \pi}{4}}\right),
$$

All the parameters are standard for light description. Emphasis must be placed on the azimuthal index $m$, since $m=0$ describes a Gaussian beam and a $m=1$ describes a Laguerre beam with an optical vortex. Figure $2 b$ illustrates the electric field norm distribution over the antenna space that can be used to justify some of the limitations of the antenna. The intensity distribution is zero at the axis of propagation $\mathrm{z}$ and changes along with the antenna radial distance and with the beam radius. An incident beam with a radius of one micrometer accommodates the whole antenna in the low-intensity central region.

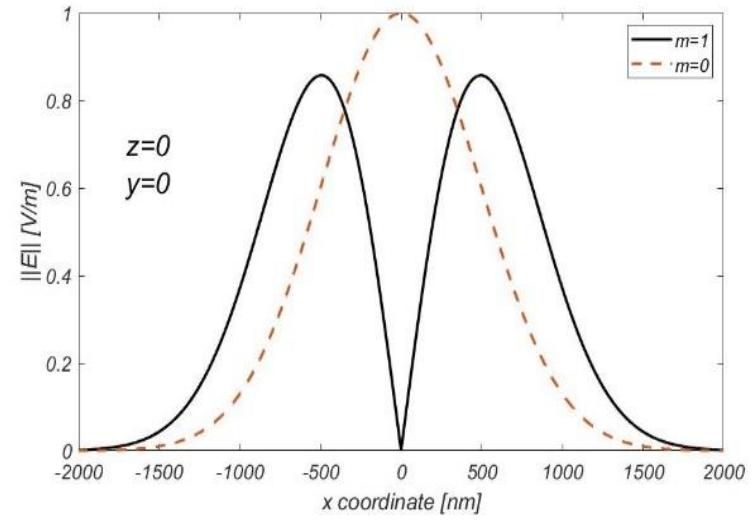

(a)

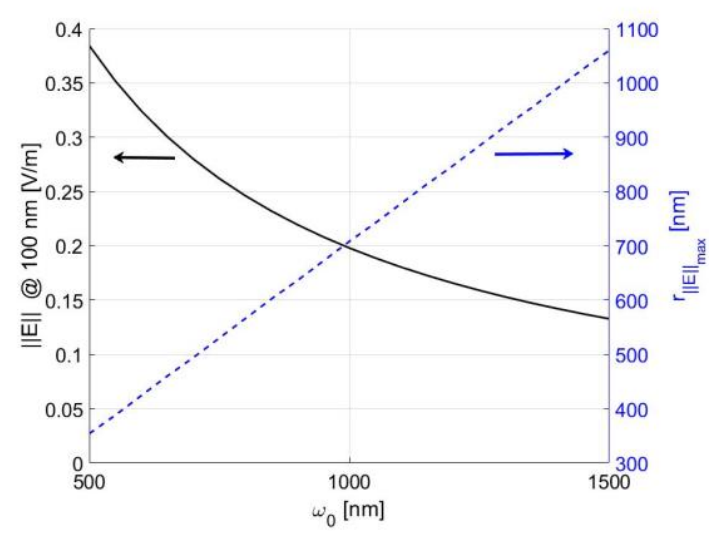

(b)

Figure 2. a) Cross-section profile of the electric field norm for a Gaussian beam $(m=0)$ and Laguerre- Gauss beam $(m=$ 1). Both beams have $\omega_{0}=700 \mathrm{~nm}$. (b) Electric field norm at $r=100 \mathrm{~nm}$, near the center of the antenna and spatial location of the maximum beam intensity. Both numbers are a function of the beam radius at the focal plane.

\subsection{The nanolens}

The plasmonic antenna relies on the dielectric function and requires a small resistivity. The accepted model for the dielectric function in metals is the Lorentz-Drude model [12], which depends on the plasmonic frequency $\left(\omega_{p}\right)$ and the dissipation factor $(\gamma)$.

$$
\varepsilon(\omega)=1-\sum_{n} \frac{\omega_{p n}^{2}}{\omega^{2}+i \omega \gamma_{n}} \approx 1-\frac{\omega_{p}^{2}}{\omega^{2}}+i \frac{\omega_{p}^{2} \gamma}{\omega^{3}}
$$

The spectral study on frequency $\omega$ requires this dependence in the dielectric function. In this work, we used parameters that are similar to gold [13] and useful for frequencies larger than $500 \mathrm{~nm}$. Figure 3 presents this dielectric function where the real part is negative, and relations with the optical constants are $\varepsilon(\omega)=\varepsilon_{1}+i \varepsilon_{2}=(n+i \kappa)^{2}$ and $n^{2}=$ $\left(|\varepsilon|+\varepsilon_{1}\right) / 2, \kappa^{2}=\left(|\varepsilon|-\varepsilon_{1}\right) / 2$. 


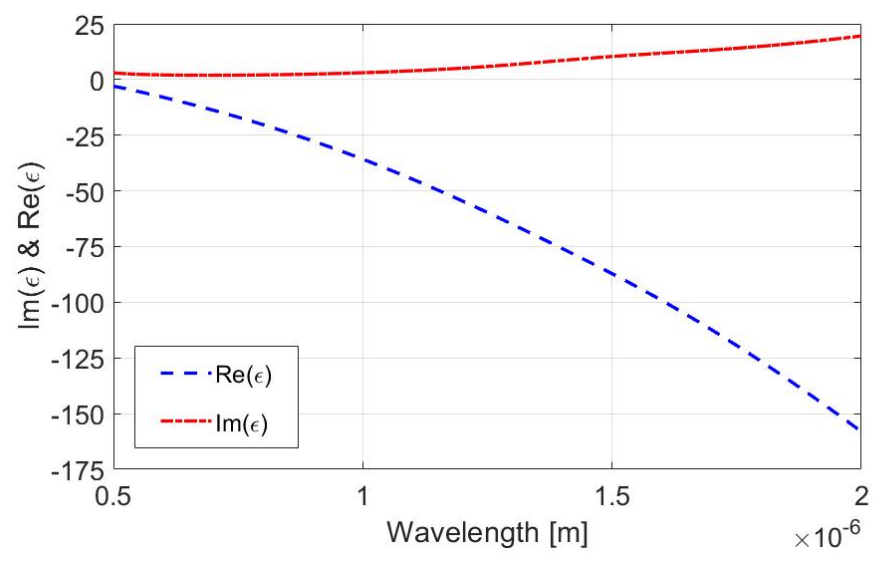

Figure 3. The dielectric function used for high conductivity metal antenna, the Lorentz-Drude model corresponds with gold.

The antenna consists of 8 arms straight rods, approximately $500 \mathrm{~nm}$ in length, $40 \mathrm{~nm}$ width and $50 \mathrm{~nm}$ in thickness [14] shown in Figure 4a. Figure 4b presents one arm, as well as its location (100 $\mathrm{nm}$ with respect to the center of the antenna) and the angle $\alpha$ between the propagation vector and the normal to the antenna plane.

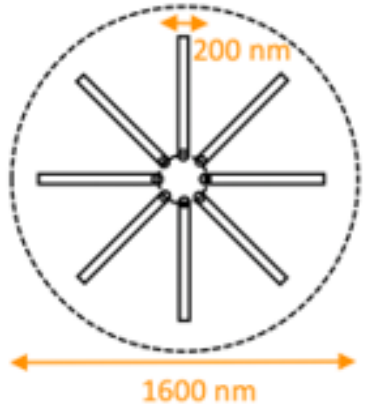

(a)

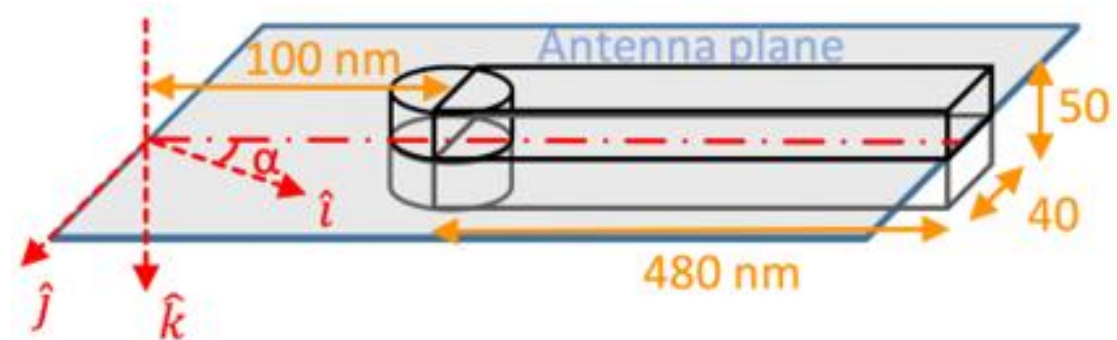

(b)

Figure 4. (a) Antenna plane with the eight arms in addition to the simulation volume cross-section with a diameter of 1600 $\mathrm{nm}$. (b) One-arm antenna geometry and antenna plane angle $(\alpha)$ relative to the electromagnetic wave propagation direction.

Figure 4a presents the geometry of the whole antenna, designed to be resonant with a wavelength of $1800 \mathrm{~nm}$ [15], and the simulation volume, where the propagation of the beam as well as the contribution from the antenna are measured.

This spatial simulation allows us to study performance as a function of the wavelength [16], and the modifications due to noncollinear conditions between the antenna axis and the propagation vector.

When the antenna plane is normal to the beam propagation, the electric field on the antenna is symmetrical if an even number of arms is used in the antenna. Otherwise, the antenna plane is not normal to the beam propagation, the electric field on the antenna is not symmetrical, though the intensity is still symmetrical. In the latter case, the adjustments to the antenna due to changes in intensity are inevitable, such as uneven arms and asymmetrical location.

\section{Results}

\subsection{Electric field control with lens}


The system performance, including the details of the Laguerre-Gauss beam, the plasmonic antenna, and the electric field profile [17] can be analyzed by finite-difference timedomain (FDTD) [14] and by finite element implemented in Mathematica $₫$ or COMSOL ®. Here the volume was segmented with an adaptable mesh, 2.2 million degrees of freedom, and 100 thousand scattered independent points that produce $2 \mathrm{~nm}$ resolution at critical locations and $100 \mathrm{~nm}$ where it is not needed.

The results of the simulation are a matrix of $x, y, z$ locations and $E_{x}, E_{y}, E_{z}$ complex numbers for each location. All the illustrations present $\operatorname{norm}(E) \equiv\|E\|$ which is produced by the source and modified by the antenna and keeping in mind do not destroy the phase profile of the beam.

\subsection{Two-arms dipole antenna intuition}

The behavior and the antenna merit depend on the light beam. To gain some intuition and visualization (case of normal incidence and Laguerre $m=1$ beam), we constructed a two-arms dipole antenna with a geometry of two $500 \mathrm{~nm}$ rods and a gap of $100 \mathrm{~nm}$, which produces the main resonance with light at approximately $1450 \mathrm{~nm}$. When we increased the gap to $200 \mathrm{~nm}$, the antenna's resonance changed to approximately $1550 \mathrm{~nm}$. The above example produces a basic guide for resonance at a wavelength approximately equal to the gap length plus 1.35 metallic length.

Figure 5 illustrates the electric field norm near the metallic rod for a linear antenna with two metallic rods $500 \mathrm{~nm}$ long and a $200 \mathrm{~nm}$ air gap. Figure 5a shows an antenna in the plane normal to the beam propagation. The black line describes the incident electric field and the blue line illustrates the produced electric field with a wavelength of $1550 \mathrm{~nm}$ incident beam, corresponding with the main resonance. The green line is produced with a wavelength of $1050 \mathrm{~nm}$, close to antiresonance, and the red line is produced with a wavelength of $790 \mathrm{~nm}$ incident beam, close to the second resonance. All the lines are symmetrical to the center of the antenna. Figure $5 b$ shows the same linear antenna in a 45 -degree slanted plane; the blue line is the field produced by a beam with a wavelength of $1600 \mathrm{~nm}$ and it is not matching the symmetrical profile. The red and blue lines show the effect of shifting the antenna by100 $\mathrm{nm}$ in the $y$-direction (positive and negative, respectively). The positive shift helps to regain the symmetry of the field profile. The cyan and magenta lines show the effect of moving the antenna in the $z$-direction (positive and negative). Here, the negative movement helps the symmetry.

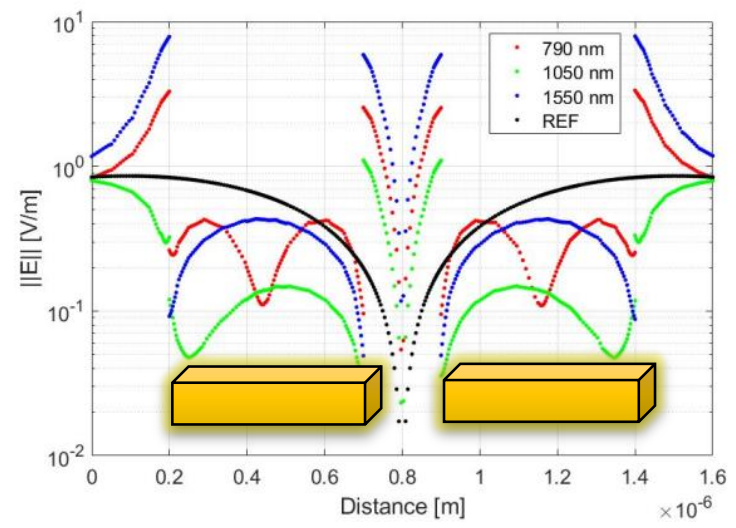

(a)

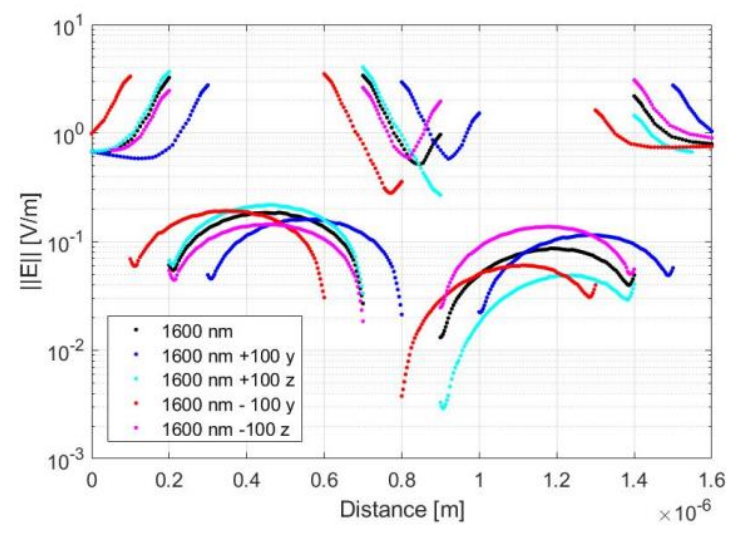

(b)

Figure 5. (a) Linear antenna in a plane with the incident beam; the black line describes the incident electric field, and a blue line illustrates the electric field produced with a wavelength of $1550 \mathrm{~nm}$ incident beam, corresponding with the main resonance. The green line is produced with a wavelength of $1050 \mathrm{~nm}$ close to antiresonance, and the red line is produced with a wavelength of $790 \mathrm{~nm}$ incident beam close to the second resonance. (b) Linear antenna at 45 degrees, slanted plane; the black line is the field produced by a beam with a wavelength of $1600 \mathrm{~nm}$ and it is not a symmetrical profile, red and blue lines are the effect of shifting the antenna $100 \mathrm{~nm}$ in the $y$-direction, a positive movement helps the symmetry. Cyan and magenta lines are the effect of moving the antenna in $z$ directions, negative movement also helps the symmetry. 


\subsection{Eight-arms antenna: Corral.}

Figure 6 presents the figure of merit of the antenna, the intensity near the center of the antenna, and the intensity along a circular $75 \mathrm{~nm}$ radius path compared to the intensity with no antenna. The blue line shows the results for normal incidence and highlights the enhancement due to arms antenna matching lambda $(\sim 1800 \mathrm{~nm})$, lambda over two ( $\sim 900$ $\mathrm{nm})$, and lambda over three $(\sim 600 \mathrm{~nm})$. Due to the symmetry, the results are produced in part for the 1D (colinear arms) and the 2D proximity of adjacent arms. The black line around the main resonant peak corresponds to the same antenna slanted at 22.5 degrees, which is associated with the blurred gray shadows indicating loss of symmetry in the electric field to the antenna lines and subsequently a variation in the intensity as indicated by the gradual gray shadow. The green line indicates the results for a 45-degree angle, and the blurred area indicates the intensity variation, which is quite severe in this case, making limited use as an intensity homogenous amplifier at this angle for this frequency region and even more severe at the other resonances.
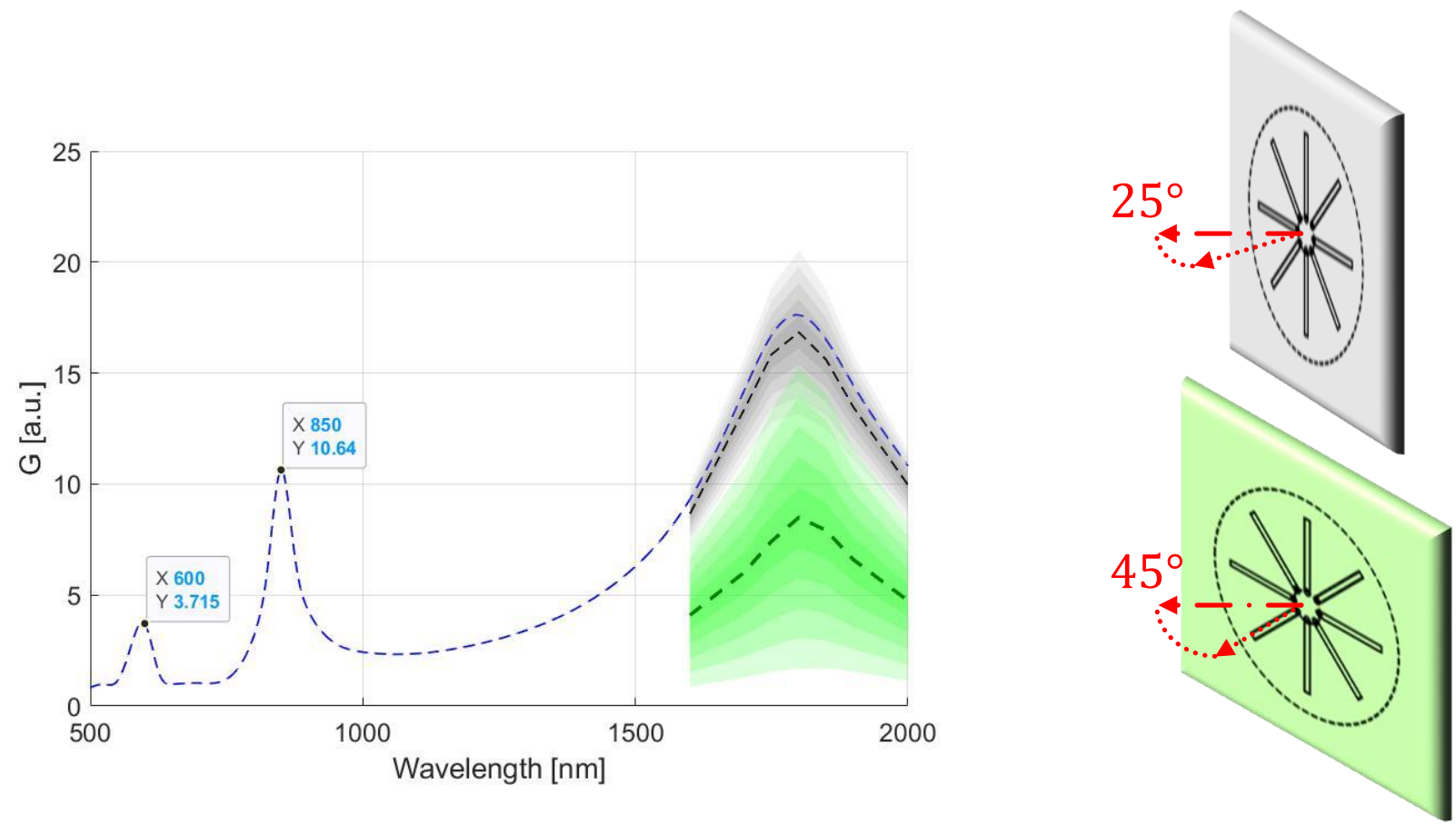

Figure 6. The figure of merit for the reference antenna, and its performance as a function of the angle between the propagation vector and antenna plane. Blue represents the normal incidence; black is 22.5 degrees and green is 45 degrees. The blurred area illustrates the gain variability where the inset color (gray and green) show the corresponding angle between the propagation vector and antenna plane.

The variability in the field enhanced corral is detailed in the supplementary material, where Figure 7 coincide with $S 4$ and describes the norm of the electric field in a polar plot at $\rho=70 \mathrm{~nm}$ from the propagation axis. The symmetrical black dots are for normal incidence to the antenna and the others are for different locations of the slanted antenna center making an electric field corral distorted with larger variation in the |EI. 


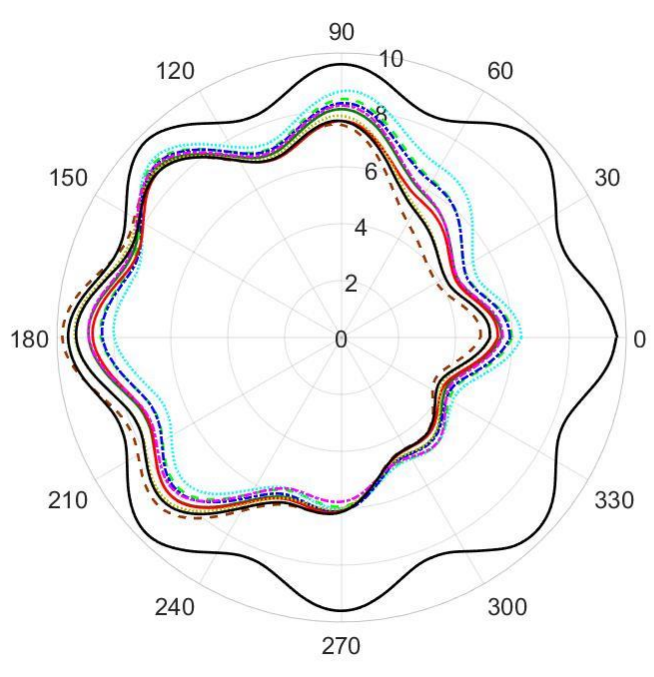

(a)

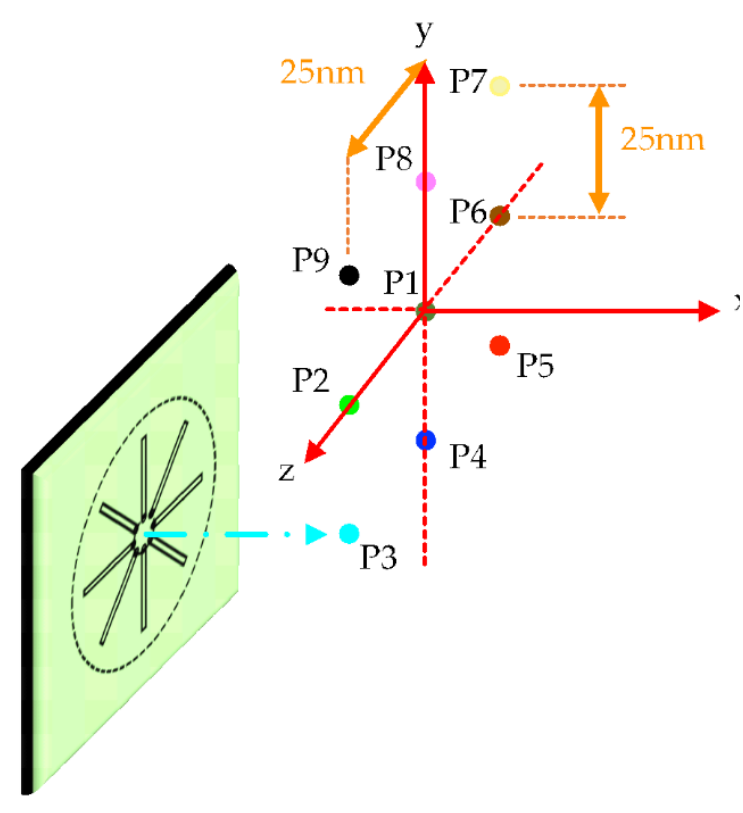

(b)

Figure 7. Contour-Polar plot for the gain variability at $70 \mathrm{~nm}$ from the centre and antenna center location, simulating defocusing with step displacement adjustments. (a) Norm of the total electric field at $\mathrm{Q}=70$. The black REF line is for normal incidence and other colors are for 45 degrees tilted antenna with position adjustments. (b) The symmetrical $x$-axis corresponds to normal incidence; the colored position adjustments are also considering the 45 degrees tilted antenna. The best conditions is for $\mathrm{y}=-5 \mathrm{~nm}$ and $\mathrm{z}=+25 \mathrm{~nm}$, the worst is for $\mathrm{y}=+25 \mathrm{~nm}$ and $\mathrm{z}=-25 \mathrm{~nm}$.

\subsection{Improve illumination with oblique incidence}

The reason for such intensity variation is the combination of the $1 \mathrm{D}$ and $2 \mathrm{D}$ structure of the antenna. The 1D colinear two arms antenna has an enhancement of the electric field at the gap of the antenna and the external extremes of the antenna (figure 5a). This enhancement is symmetrical in the electric field due to the symmetry in the antenna and the incident field preserving the incident beam properties. If the linear antenna is aligned with the slanted plane, the electric field enhancement loses its symmetry, which is readily noticeable because it erases the zero intensity in the antenna center. The 2D structure demands symmetry with the incident field that a normal antenna readily provides. On the other hand, a slanted antenna exposes each arm to a different electric field and phase, which enhances the properties of the incident beam more difficult to preserve.

To compare the symmetry of field enhancement at the center of the antenna, a metric needs to be defined: the gain variation along a circular path near the antenna center, a radius $\rho<80 \mathrm{~nm}$ is free from the antenna. A uniform enhancement antenna will have a minimum gain variation. To decrease the gain variation along the path, a combination of (1) antenna defocusing (z-axis movement), (2) antenna misalignment ( $x$ and y movement), see table TS1 in the supplementary material and (3) a simple antenna redesign changing rods length is proposed. For example, increasing the length of one antenna does not produce a measurable advantage, see supplementary material figures S9 to S16. However, a clear advantage is seen with the reduction of one arm, see figures S17 to S24.

Changing the antenna in a minute amount $(30 \mathrm{~nm})$ goes in both directions; fabrication variability may dominate a specific design linked to good performance, however, opens the door to seek and try intentional variations in the design. Figures S26 and S28 in the supplementary material illustrate such possibility, with three arms $30 \mathrm{~nm}$ shorter at $90^{\circ}$, $135^{\circ}$, and $180^{\circ}$, and a noticeable improvement in the variability was identified. 
Figure 8 illustrates the electric field norm, which is equal to the gain because it is normalized to the incoming beam, for an (LG) $m=1$ beam over the antenna at normal incidence, where the symmetry helps to visualize the first three resonant frequencies $(8 \mathrm{a})$, and the gain is largest for the first resonance. $8 \mathrm{~b}$ is for a $22.5^{\circ}$ slanted antenna y $8 \mathrm{c}$ is for a $45^{\circ}$ slanted antenna, in these last two cases, the loss of symmetry is evident as illustrated by the intensity in the corral.
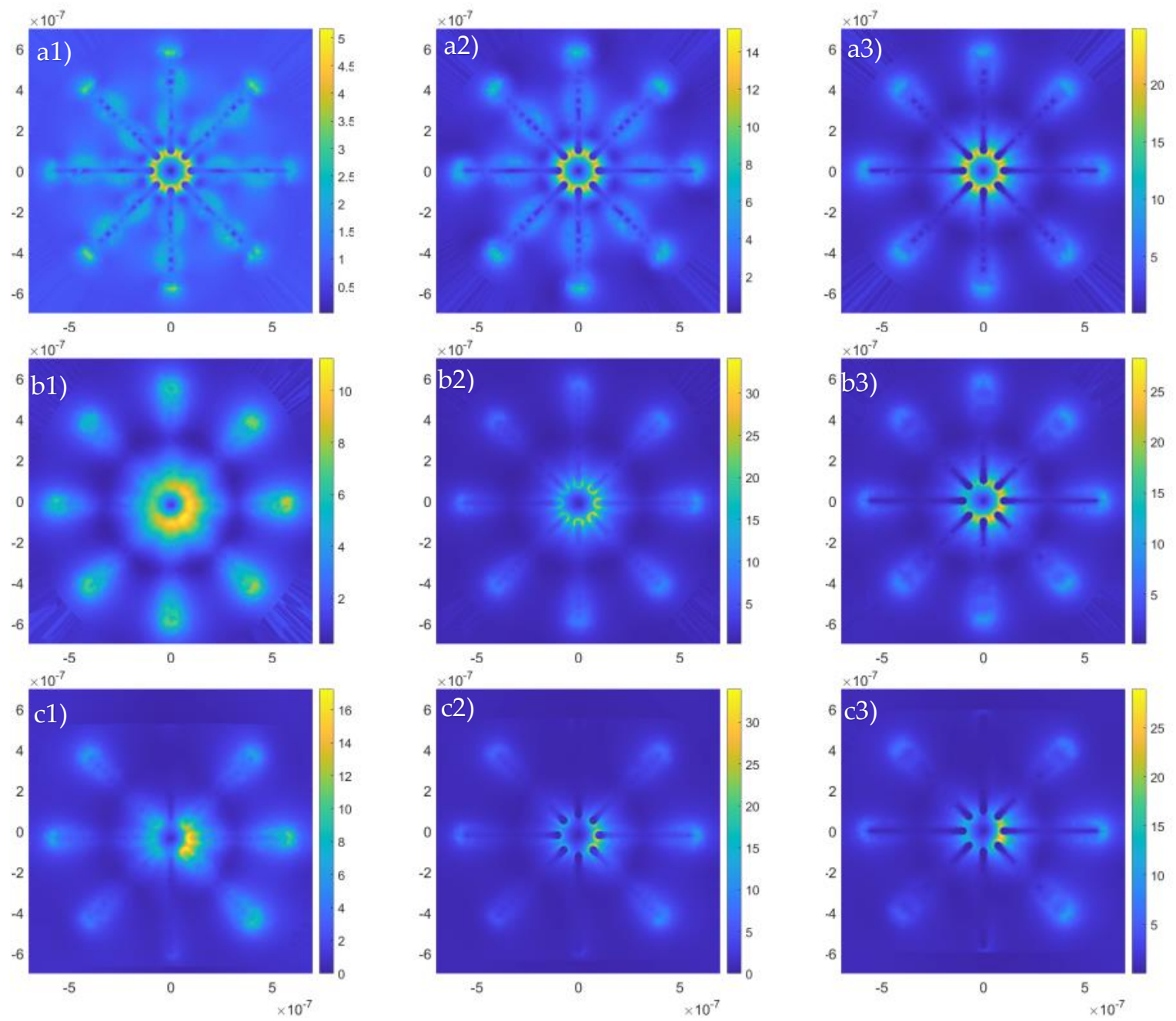

Figure 8. a) Gain field for a beam at normal incidence to the antenna with an (LG) $m=1$ at $600 \mathrm{~nm}, 850 \mathrm{~nm}$, and 1800 $\mathrm{nm}$ wavelength excitation, for label a1, a2, or a3 respectively. b) Gain field for incidence at $22.5^{\circ}$ to antenna $1800 \mathrm{~nm}$ at a plane $50 \mathrm{~nm}, 30 \mathrm{~nm}$, and $0 \mathrm{~nm}$ over antenna plane, for label b1, b2, or b3 respectively. c) Gain field with an incident beam (LG) $m=1$, at $45^{\circ}$ respect to antenna $1800 \mathrm{~nm}$ at a plane $50 \mathrm{~nm}, 30 \mathrm{~nm}$, and $0 \mathrm{~nm}$ over antenna plane, for label c1, c2, or c3 respectively.

\section{Discussion}

To realize a plasmonic antenna interacting with light carrying orbital angular momentum, in our case for an LG beam, faces many obstacles[18]; for normal incidence, the antenna size, the beam size, and the working wavelength demand conflicting requirements[19]. To work with larger antennas, one needs to work with higher resonances, this is in addition to the diminished properties of the dielectric constant at short wavelengths [20]. An antenna designed for $532 \mathrm{~nm}$ may have such a small footprint that will be exposed to the center of the beam and consequently to a very small electric field. Figure 8a illustrate the enhanced intensity profile at the first three stationary waves that match the three peaks in figure 6, the label a1), a2) and a3) correspond to 600, 850, and $1800 \mathrm{~nm}$ respectively, the 
intensity is better appreciated in figure 6 , the symmetry is shown in figure 8 . Figure $8 \mathrm{~b}$ shows the gain profile at $1800 \mathrm{~nm}$ (first resonance) for incidence at 22.5 degrees, at three planes parallel to the antenna, 50,30, and $0 \mathrm{~nm}$ meaning the defocusing with labels b1), $\mathrm{b} 2$ ), and b3) respectively. In those images the horizontal axis (the $x$-axis) is used to rotate the antenna, the top part (positive $y$ ) is now at positive $z$ and the lower part (negative $y$ ) is at negative $z$. The image can be misleading if compared, due to different colors for the same intensity. Figure 8c presents pictures for the gain profile at $1800 \mathrm{~nm}$ for incidence at 45 degrees at three planes parallel to the antenna, 50, 30, and $0 \mathrm{~nm}$ with labels c1), c2) and c3) respectively. In those images the horizontal axis (the $x$-axis) is used to rotate the antenna, the top part (positive $y$ ) is now at positive $z$ and the lower part (negative $y$ ) is at negative $z$. The intensity in the image should not be taken as a guide for the rotation.

This immediately shows the limitation of antennas at angles different from normal incidence and gives some knowledge of how to construct the antenna for such angles. Two factors determine the efficiency of the antenna, usually conflicting: (1) the electric field magnitude over the antenna, because (LG) beams maximum gets further from the center increasing the beam diameter (Fig. 2b), though the working frequency decreases for the same reason, and (2) the rod length and central gap structure in the dipole antenna defines the resonant frequency. In a multi-rod antenna, attempts to correct the slanted angle $\alpha$ are limited because the antenna sees a beam growing from 1 to $1 / \cos (\alpha)$ in the two normal axes and it is already doubling for $\alpha$.

From the systematic results presented in the supplementary material (S17 to S24), a reduction in one arm's length is advantageous to compensate for the slanted angle, and a combination of lengths is even more convenient presenting the possibility of partially correcting the effects of the slanted angle.

\section{Conclusions}

We have discussed the effect of oblique illumination on the functioning of a plasmonic nanoantenna for chiral light. The objective of focusing the intensity profile of the electric field in a LG, $m=1$ beam, while preserving its properties, is achievable with a nanoantenna. If the beam symmetry is available at the antenna plane, relatively simple antenna geometry and alignment are needed, scaled for the wavelength used, considering that the dielectric effect allows, as described in figures $2 b$ and 3 . When a slanted antenna and an LG, $m=1$ is available, adjustments in the distance between the beam focal plane and the antenna plane, as well as the position of the beam axis to the antenna axis will be needed as suggested in figure 5, and the antenna needs to be designed by decreasing appropriately the arm's length following the vortex structure in a way that increases the antenna's usability. The study also has shown that orbital angular momentum manipulation is possible with tilted light incidence giving rise to a controlled intensity distribution, the interaction beam phase front, and the effects of possible tunable OAM with the nanoantenna's geometry, that opens the door to control properties and optical manipulation technologies for advanced application [21].

Supplementary Materials: The following are available online at www.mdpi.com/xxx/S1_Supplementary Material.

Author Contributions: All authors contributed equally to this work.

Funding: RQT acknowledges the support from UNAM-DGAPA PASPA. We thank the Robert A. Welch Foundation (Award \# A-1547), and the Texas A\&M Triads for Transformation program. JLDJ thanks Catedras CONACYT-UNAM. R.Q.-T and JLDJ wish to thank the financial support from CONACYT, under grant A1-S-8317.

Data Availability Statement: Data is co

Acknowledgments: Special thanks should be given to Dr. Jose Luis Aragon Vera as a research project supervisor of CATEDRAS-CONACYT grant number 1039 in the Centro de Física Aplicada y Tecnología Avanzada at the UNAM. 
Conflicts of Interest: The authors declare no conflict of interest.

\section{References}

1. H. Yu, Y. Peng, Y. Yang, and Z. Y. Li, "Plasmon-enhanced light-matter interactions and applications," npj Comput. Mater. (2019).

2. J. Wyrick, X. Wang, R. V. Kashid, P. Namboodiri, S. W. Schmucker, J. A. Hagmann, K. Liu, M. D. Stewart, C. A. Richter, G. W. Bryant, and R. M. Silver, "Atom-by-Atom Fabrication of Single and Few Dopant Quantum Devices," Adv. Funct. Mater. (2019).

3. A. R. Kraemer and R. W. Jones, "Optical Antennas.," Int. Telemetering Conf. 14, 347-356 (2009).

4. A. E. Krasnok, I. S. Maksymov, A. I. Denisyuk, P. A. Belov, A. E. Miroshnichenko, C. R. Simovski, and Y. S. Kivshar, "Optical nanoantennas," Physics-Uspekhi 56, 539-564 (2013).

5. D. Naidoo, F. S. Roux, A. Dudley, I. Litvin, B. Piccirillo, L. Marrucci, and A. Forbes, "Controlled generation of higher-order Poincaré sphere beams from a laser," Nat. Photonics 10, 327-332 (2016).

6. A. Holleczek, A. Aiello, C. Gabriel, C. Marquardt, and G. Leuchs, "Classical and quantum properties of cylindrically polarized beams of light via a poincaré sphere representation," Opt. InfoBase Conf. Pap. 19, 9714-9736 (2011).

7. M. Shutova, A. D. Shutov, and A. V. Sokolov, "Spectroscopic sensing enhanced by quantum molecular coherence and by plasmonic nanoantennas," Proc. SPIE 11296 1129605, 4 (2020).

8. A. Afanasev, C. E. Carlson, C. T. Schmiegelow, J. Schulz, F. Schmidt-Kaler, and M. Solyanik, "Experimental verification of position-dependent angular-momentum selection rules for absorption of twisted light by a bound electron," New J. Phys. (2018).

9. C. T. Schmiegelow, J. Schulz, H. Kaufmann, T. Ruster, U. G. Poschinger, and F. Schmidt-Kaler, "Transfer of optical orbital angular momentum to a bound electron," Nat. Commun. (2016).

10. K. A. Forbes and D. L. Andrews, "Spin-orbit interactions and chiroptical effects engaging orbital angular momentum of twisted light in chiral and achiral media," Phys. Rev. A (2019).

11. W. N. Plick and M. Krenn, "Physical meaning of the radial index of Laguerre-Gauss beams," Phys. Rev. A - At. Mol. Opt. Phys. 92, 1-10 (2015).

12. E. D. Palik, Handbook of Optical Constants of Solids (2012).

13. A. D. Rakić, A. B. Djurišić, J. M. Elazar, and M. L. Majewski, "Optical properties of metallic films for vertical-cavity optoelectronic devices," Appl. Opt. (1998).

14. R. W. Heeres and V. Zwiller, "Subwavelength focusing of light with orbital angular momentum," Nano Lett. 14, 4598-4601 (2014).

15. L. Novotny and N. Van Hulst, "Antennas for light," Nat. Photonics 5, 83-90 (2011).

16. P. Ghenuche, S. Cherukulappurath, T. H. Taminiau, N. F. Van Hulst, and R. Quidant, "Spectroscopic mode mapping of resonant plasmon nanoantennas," Phys. Rev. Lett. 101, 1-4 (2008).

17. L. Novotny, "Optical Antennas: A New Technology that can Enhance Light-Matter Interactions," Front. Eng. 39, 100-120 (2012).

18. Hachtel, J.A., Cho, SY., Davidson, R.B. et al. Spatially and spectrally resolved orbital angular momentum interactions in plasmonic vortex generators. Light Sci Appl 8, 33 (2019).

19. Kerber, R. M.; Fitzgerald, J. M.; Reiter, D. E.; Oh, S. S.; Hess, O. Reading the Orbital Angular Momentum of Light Using Plasmonic Nanoantennas. ACS Photonics, 4, 891- 896, (2017).

20. Takashi Arikawa, Shohei Morimoto, and Koichiro Tanaka, "Focusing light with orbital angular momentum by circular array antenna," Opt. Express 25, 13728-13735 (2017).

21. Shen, Y., Wang, X., Xie, Z. et al. Optical vortices 30 years on: OAM manipulation from topological charge to multiple singularities. Light Sci Appl 8, 90 (2019). 